\title{
Tratamiento multisistémico en adolescentes con trastorno disocial
}

Francisco $R$ de la Peña-O Ivera, MC, M en C.(1)

\section{De la Peña-Olvera FR. \\ Tratamiento multisistémico \\ en adolescentes con trastorno disocial. Salud Publica Mex 2003;45 supl 1:S124-S131.}

\section{Resumen}

El presente trabajo muestra las generalidades del trastorno disocial, así como las principales medidas para su reconocimiento y tratamiento. Aborda las generalidades y principios básicos de la terapia multisistémica enfocada para este trastorno; dicha terapia se basa en intervenciones bioló gicas, psicológicas y sociales, y considera el ámbito ecológico de las manifestaciones de la conducta de adolescentes. Se muestran algunos resultados en la aplicación de la terapia y se discuten sus ventajas y usos potenciales.

Palabras clave: terapia multisistémica; adolescente; trastorno disocial

\section{De la Peña-Olvera FR. Multisistemic therapy of adolescents with conduct disorder. Salud Publica Mex 2003;45 suppl 1:S124-S131.}

\section{A bstract}

This paper reviews the main features of conduct disorder (CD), as well as the principles for diagnosis and multisystemic treatment (MST). MST includes biological, psycholo gical and social interventions, and considers the ecological environment of conduct manifestations. Some outcomes of MST delivery are discussed, along with its advantages and potential applications.

Key words: multisystemic treatment; adolescent; conduct disorder
$\mathrm{L}$ a intención en este artículo es abordar el tratamiento multisistémico de adolescentes con trastorno disocial. En la primera parte planteamos el marco teórico y conceptual de la categoría diagnóstica denominada trastorno disocial (TD), abordamos los criterios taxonómicos internacionales, el curso de la en- fermedad, los subtipos del padecimiento, la evaluación de los síntomas, las correlaciones clínicas con el consumo de alcohol y drogas, los factores de riesgo y las opciones de tratamiento tanto biológicas como psicosociales. Posteriormente, en la segunda parte hablamos de la intervención psicosocial de nuestro interés: el

(1) Departamento de Psicología Médica, Psiquiatría y Salud Mental de la Facultad de Medicina de la Universidad N acional Autónoma de México, México, D.F., México. Clínica de Adolescentes del Instituto N acional de Psiquiatría Ramón de la Fuente Muñiz. México, D.F.

Fecha de recibido: 18 de abril de 2001 • Fecha de aceptado: 18 de junio de 2002 Solicitud de sobretiros: Dr. Francisco R de la Peña-O Ivera, Calzada México-Xochimilco 101, colonia San Lorenzo Huipulco, 14370,Tlalpan, México, D.F., México.

Correo electrónico: adolesc@imp.edu.mx 
tratamiento multisistémico (TMS), del cual señalamos sus fundamentos teóricos así como sus bases filosóficas, y delineamos los aspectos generales de la aplicación clínica básica basándonos en los principios de esta alternativa terapéutica. Finalmente, se busca presentar los resultados de investigaciones desarrolladas con la TMS y una conclusión sobre la misma en adolescentes con TD, sus beneficios y usos potenciales.

\section{El trastorno disocial}

El trastorno disocial se considera una categoría diagnóstica médico psiquiátrica codificada tanto en el Manual Diagnóstico y Estadístico de los Trastornos Mentales (DSM) de la Asociación Psiquiátrica Americana (APA); ${ }^{1}$ como en la Clasificación Internacional de Enfermedades (CIE) de la Organización Mundial de la Salud (OMS) ${ }_{;}^{2}$ los TD en la CIE 10 abarcan la siguientes categorías diagnósticas (F91): TD limitado al contexto familiar, TD en niños no socializados, TD en niños socializados, TD desafiante y oposicionista, otros trastornos disociales y trastorno disocial sin especificación. Un grupo cada vez mayor de investigadores denomina al conjunto de tres categorías diagnósticas del DSM-IV: ${ }^{1} \mathrm{TD}$, trastorno desafiante oposicionista (TDO) y trastorno por déficit de atención con hiperactividad (TDAH) como trastornos exteriorizados. En el cuadro I presentamos los criterios diagnósticos para el TD de acuerdo con el DSM-IV, ${ }^{1}$ esta categoría taxonómica de la APA es más utilizada en las investigaciones tanto clínicas como epidemiológicas que las categorías de la OMS. ${ }^{3}$ Una de las principales limitantes de estas aproximaciones diagnósticas es incluir un conjunto muy variado de síntomas que pueden ir desde la mentira hasta el herir a otro con un arma, lo que convierte a este grupo en uno muy heterogéneo. ${ }^{4} \mathrm{Al}$ dividir en subgrupos este padecimiento se reduce la heterogeneidad, por lo que algunos investigadores lo han hecho dependiendo de si la conducta es grupal o solitaria, o si el inicio de los problemas de conducta fue en la infancia o en la adolescencia; se ha podido demostrar que a menor edad de inicio, mayor severidad del padecimiento, mayor agresividad, y existen más posibilidades de que éste continúe en la vida adulta; la probabilidad de que un joven siga teniendo problemas de conducta en la vida adulta, y éstos se manifiesten como un trastorno antisocial de la personalidad (TAP), es doble cuando el problema de conducta empezó en la infancia. ${ }^{5}$ El padecimiento ha estado vinculado de forma estrecha con la violencia ya que aproximadamente $80 \%$ de los jóvenes detenidos por infracciones o delitos violentos cursan con este trastorno..$^{6-8}$ Los rangos en los reportes de la prevalencia del TD van desde

\section{Cuadro I \\ Criterios diagnósticos del trastorno disocial-IV}

A. Un patrón repetitivo y persistente de comportamiento en el que se violan los derechos básicos de otras personas o normas sociales importantes propias de la edad, manifestándose por la presencia de tres o más de los siguientes criterios durante los últimos 12 meses y por lo menos de un criterio durante los últimos seis meses.

Agresión a personas y animales

1. A menudo fanfarronea, amenaza o intimida a otros

2. A menudo inicia peleas físicas

3. Ha utilizado un arma que puede causar un daño físico grave a otras personas (palo, botella, navaja, pistola)

4. Ha manifestado crueldad física con personas

5. Ha manifestado crueldad física con animales

6. Ha robado enfrentándose a la víctima

7. Ha forzado a alguien a una actividad sexual

Destrucción de la propiedad

8. Ha provocado deliberadamente incendios con la intención de causar daños graves

9. Ha destruido deliberadamente propiedades de otras personas (distinto de provocar incendios)

Fraudulencia y robo.

10. Ha violentado el hogar, la casa o el automóvil de otra persona

11. A menudo miente para obtener beneficios, favores 0 evitar obligaciones

12. Ha robado objetos de cierto valor sin enfrentar a la víctima

Violaciones graves de normas

13. A menudo permanece fuera de casa de noche a pesar de las prohibiciones paternas, iniciando este comportamiento antes de los 13 años de edad

14. Se ha escapado de casa por la noche por lo menos en dos ocasiones (o sólo una vez si tarda mucho en regresar)

15. Suele hacer novillos o irse de pinta de la escuela, e inicia esta práctica antes de los 13 años

B. El trastorno disocial provoca deterioro clínicamente significativo de la actividad social, académica o laboral

C. Si el individuo tiene más de 18 años no debe cumplir con los criterios del trastorno antisocial de la personalidad

Debe especificarse el tipo de inicio, infantil ( $<10$ años) 0 adolescente (>10 años)

Debe especificarse la gravedad: leve (pocos problemas de comportamiento y sólo causan daños mínimos a otros; moderado (intermedio entre leve y grave); grave (varios problemas del comportamiento y se causan daños serios a otros)

1 a $10 \%{ }^{3}$ en poblaciones abiertas de niños y adolescentes, y hasta $28 \%$ en muestras clínicas; esta variabilidad está condicionada por la forma de evaluar el padecimiento (evaluación clínica vs entrevista diagnóstica) por la definición operacional establecida (DSMIII-R vs DSM-IV) y por el grupo seleccionado (hombres vs mujeres). La relación hombre-mujer en el TD es de 4:1. La evaluación de la sintomatología del TD exige que el clínico conozca los criterios operacionales del padecimiento y utilice un instrumento diagnóstico estandarizado; las entrevistas clínicas que pueden uti- 
lizarse son la entrevista semiestructurada para adolescentes ${ }^{9}$ y la Entrevista Semiestructurada Kiddie Schedule for affective disoreders and schizophrenia PL (K-SADS-PL); ${ }^{10}$ estos instrumentos permiten al clínico obtener de una forma racional, organizada y sistematizada los síntomas del padecimiento en el episodio actual y en el pasado; los coeficientes de correlación tanto interevaluador como temporal para el TD se han reportado con $\mathrm{K}>0.80$ en ambas entrevistas. Asimismo se han utilizado escalas de tamizaje o severidad como el Child Behavior Check List (CBCL) ${ }^{11}$ y el cuestionario de conducta para padres, niños y adolescentes de Conners; ${ }^{12}$ estas entrevistas y escalas permiten establecer diagnósticos de certeza y llevar el seguimiento de los síntomas durante el tratamiento.

\section{El trastorno disocial: el alcohol y las drogas}

Probablemente una de las correlaciones clínicas más importantes con el TD sea la de adolescentes que consumen alcohol o drogas. Más de $60 \%$ de los adolescentes con TD tienen algún tipo de abuso o dependencia a alcohol o drogas. ${ }^{13}$ Las siguientes características conductuales son propias de adolescentes que abusan o dependen de las sustancias: impulsividad, agresividad, búsqueda de sensaciones, baja evitación al daño, incapacidad para postergar gratificaciones, falta de religiosidad y psicopatología, en especial el TD. ${ }^{14,15}$ Los mecanismos que se han propuesto para explicar la relación entre el consumo de alcohol o drogas y los actos disociales son: 1) un crimen violento es un camino habitual para obtener la droga; 2) la violencia (amenazas, golpes, heridas) puede ser una condición general para resolver las disputas entre quienes distribuyen las drogas; 3) el uso de sustancias y la conducta iracunda pueden tener factores causales similares y por lo tanto concurrir en determinados sujetos, y 4) ciertas sustancias incrementan la probabilidad de presentar conducta violenta, situación que se explica por sus propiedades farmacodinámicas (sus mecanismos y sitios de acción en el organismo). ${ }^{16}$ A continuación mencionamos los principales efectos de algunas drogas con la conducta disocial en adolescentes. Alcohol: los reportes médico legales señalan que al menos $62 \%$ de los criminales violentos consumieron etanol poco antes de perpetrar el delito por el cual fueron encarcelados. El alcohol incrementa la agresividad, especialmente en los varones, pero es importante mencionar que esta acción es dosis dependiente. Los estudios sugieren que las cantidades elevadas de alcohol tienen una acción analgésica y depresora sobre el sistema psicomotor, mientras que en dosis bajas favorecen la aparición de los efectos contrarios. Psicoestimulantes: se ha demostrado que las anfetaminas favorecen la competitividad y la excitabilidad, pero no existen datos concluyentes de que conduzcan a la violencia. La cocaína: incrementa la agresividad sólo a dosis altas en los individuos sanos no farmacodependientes; parece que los individuos que consumen cocaína son esencialmente más violentos que quienes no lo hacen. Cannabis: no existe correlación alguna entre las conductas disociales y la marihuana, inclusive algunos datos encontrados tanto en estudios de animales como en humanos, apuntan a que los cannabinoides reducen la agresividad; la asociación entre cannabis y agresividad, cuando se observa, debe explicarse por fenómenos no farmacodinámicos. Inhalables (cemento, activo, tinner, etcétera): existen reportes de casos de la asociación entre los inhalables y las conductas disociales, sin embargo, hasta la fecha no se han reportado estudios sistematizados que busquen la correlación con la agresividad en personas que usan frecuentemente estas sustancias. ${ }^{17}$

\section{Elementos etiológicos y factores de riesgo para el trastorno disocial}

Aunque entendemos de manera incompleta las causas precisas del TD, podemos reconocer algunos de sus factores de riesgo agrupados fundamentalmente en cuatro áreas: psicobiológica, socioeconómica, neuroendócrina y genética. A continuación algunos ejemplos de ellos. 1) Temperamento infantil: aquellos niños que demuestran respuesta con patrones oposicionistas, que tienen un elevado nivel de actividad y una baja adaptación social padecen más conductas violentas y berrinches; 2) factores parentales: se ha demostrado que la presencia de psicopatología en los padres, de conductas criminales y de una inadecuada y confusa relación paterno-filial aumentan el riesgo de TD en los niños; 3) factores familiares: se ha observado más la presencia del TD en niños de familias muy numerosas o con poca cohesión; 4) factor socioeconómico: el TD se presenta más en los niños con bajo nivel socioeconómico; 5) factores comunitarios: se ha observado mayor prevalencia del TD en comunidades con elevada criminalidad; 6) factores escolares: malas condiciones del inmueble escolar, pobre énfasis en lo académico, poco tiempo utilizado en la preparación de las clases y métodos punitivos de enseñanza,18 7) factores biológicos: se ha observado mayor prevalencia de partos traumáticos y traumatismos cráneo encefálicos en niños y adolescentes con TD, así como mayor presencia de signos neurológicos blandos; 8) factores neuroendócrinos: se ha observado un menor nivel del 
ácido 5-hidroxiindolacético y mayores niveles de testosterona en el líquido cefalorraquídeo de adolescentes con TD; se han encontrado también bajos niveles de actividad noradrenérgica en estos sujetos; 9) factores genéticos: se ha observado una mayor prevalencia de adolescentes con genotipo $47 \mathrm{XYY}$ en áreas de reclusión; en los estudios de adopción se ha identificado como mayor factor de riesgo el ser hijo de un padre disocial, y 10) abuso y negligencia: aquellos adolescentes que fueron o son víctimas de abuso físico, sexual o psicológico, o bien de negligencia por parte de los cuidadores, tienen mayor riesgo de presentar el TD. ${ }^{19}$

\section{Tratamiento}

Hasta antes de la (TMS) parecía poco alentador el tratamiento de adolescentes portadores de TD cuando se emprendían terapéuticas individuales; creemos necesario revisar algunas de las opciones que se han desarrollado en las diferentes áreas: la biológica y la psicosocial.

El carbonato de litio constituye el fármaco de primera elección en pacientes bipolares con conductas agresivas, así como en los pacientes con retraso mental y conductas violentas; los niveles terapéuticos en este último grupo se han reportado entre 0.6-1.4 $\mathrm{mEq}$./l. El principal inconveniente de este fármaco es que precisa una estrecha monitorización para evitar dosis tóxicas. El carbonato de litio ha mostrado ser efectivo en el abordaje de niños y adolescentes, tanto en estudios de tipo abierto ${ }^{20}$ como doble-ciego. ${ }^{21}$ En estos últimos se ha comprobado que el litio, a dosis medias de $1248 \mathrm{mg}$ /día es superior al placebo en la reducción de las puntuaciones en escalas de agresividad y conductas disociales, en una muestra de 50 pacientes, con una edad media de nueve años, hospitalizados por conductas agresivas severas resistentes a otros tratamientos. El litio ha demostrado su efectividad en adolescentes bipolares que abusan de sustancias. $^{22} \mathrm{Al}$ parecer el litio ha mostrado resultados inconsistentes para el control de la agresividad en niños y adolescentes, sin embargo, existen reportes sobre la utilidad del litio en el TD con sintomatología explosiva. Los antidepresivos inhibidores selectivos de la recaptura de serotonina (ISRS) han demostrado poseer una acción antiagresiva en diferentes tipos de pacientes, y no sólo en individuos depresivos, pero al contrario de lo que sucede con antisicóticos y los ansiolíticos, la acción antiagresiva de los ISRS no es atribuible a los efectos sedantes, ya que estos fármacos no están dotados de esta actividad de forma importante; la fluoxetina entre los 20 a $40 \mathrm{mg} /$ día ha demostrado su efectividad en pacientes con retraso mental y conductas agresivas y autolesivas; se ha constatado $^{23}$ que las manifestaciones de hostilidad y las reacciones antisociales tienen cuatro veces más probabilidad de presentarse en sujetos tratados con placebo que en aquellos tratados con un fluoxetina; todos los ISRS parecen ser eficaces en el manejo de las manifestaciones agresivas que acompañan a los trastornos límite de la personalidad, eficacia extensible también a la venlafaxina. Recientemente ${ }^{24}$ se realizó un ensayo abierto con ISRS (fluoxetina, paroxetina y sertralina) en una muestra de 19 adolescentes hospitalizados. Al contrario de lo que sucede con los adultos, estos agentes serotoninérgicos no fueron efectivos, 12 de los 19 pacientes incrementaron los síntomas auto y heteroagresivos durante el tratamiento. La eficacia antiagresiva de los antidepresivos clásicos es menos manifiesta. La fenelzina y la tranilcipromina, dos inhibidores de la monoaminoxidasa (IMAO), también han demostrado su eficacia en estudios controlados con pacientes diagnosticados con trastorno límite de la personalidad. ${ }^{25}$ Los antisicóticos han sido utilizados de forma amplia, pero debido a sus efectos colaterales inmediatos y a mediano y largo plazo, como el parkinsonismo, raro en prescolares, la distonia, más frecuente en adolescentes y el síndrome neuroléptico maligno no son recomendables. Sugerimos al lector interesado consultar las revisiones señaladas en las referencias. ${ }^{26-29} \mathrm{El}$ grupo de los antisicóticos atípicos como la risperidona, la clozapina y la olanzapina, parecen tener buenas expectativas en el manejo de algunos casos específicos como la etapa aguda en adolescentes con trastorno bipolar (TB), comórbido con $\mathrm{TD}$, donde dosis de hasta $4 \mathrm{mgs}$./día pueden ser necesarias, ${ }^{26,27}$ los efectos colaterales de los antisicóticos atípicos pueden ser la neutropenia, las crisis convulsivas y la hepatotoxicidad. La evaluación basal y, durante el tratamiento, son necesarios controles en la biometría hemática, pruebas de funcionamiento hepático y el electroencefalograma. La clonidina, que es un agonista alfa dos adrenérgico ha sido utilizado de forma más o menos exitosa también para el control de los síntomas agresivos y disociales.

Una de las principales intervenciones psicosociales ha sido la terapia familiar; en ésta se trata de modificar patrones agresivos de comunicación, eliminar alianzas patológicas y favorecer el desarrollo de sus miembros; en algunas ocasiones se canaliza al tratamiento específico individual a cualquiera de los miembros si se identifica como caso. La terapia cognoscitiva-conductual (TCC) ha sido la más utilizada en las diferentes investigaciones de tratamiento psicoterapéutico en adolescentes disociales; los proce- 
sos cognoscitivos de percepción, auto conocimiento, atribuciones y destrezas en la solución de problemas deben ir de acuerdo con el manejo de los roles en los problemas de conducta; estos procesos se refieren a la manera en la que el o la adolescente se enfrenta a las diferentes situaciones que vive. El adolescente muestra agresividad cuando está predispuesto a situaciones hostiles; la solución de problemas por medio de TCC consiste en el desarrollo interpersonal cognoscitivo de las destrezas para solucionar los problemas. Orientados los adolescentes hacia una mejor conducta social se les enfoca principalmente en la manera en que éstos puedan aprovechar las oportunidades; se les enseña a solucionar sus problemas interpersonales, utilizando técnicas que incluyen juegos, actividades académicas e historias; se combinan varios tipos de procedimiento como el rol playing y el reforzamiento. Se ha demostrado que este tipo de intervención puede disminuir conductas disociales. Existen también programas psicoeducativos dirigidos a los padres o cuidadores, con los cuales se les enseña sobre el TD, la eliminación de la agresividad en las relaciones familiares, los límites, la responsabilidad, y el manejo del estrés, entre otras áreas. La mayoría de las intervenciones psicosociales tienen éxito a corto plazo o cuando los ambientes son altamente estructurados, pero sus beneficios desaparecen en el mediano y largo plazo. Se han ensayado algunas estrategias como las denominadas hospitalización parcial, programas de tratamiento residencial y programas escolares especiales. ${ }^{30}$

A manera de conclusión se puede establecer que tanto los diferentes tratamientos biológicos como los psicosociales pueden controlar a corto plazo algunas manifestaciones disociales, sobre todo las más violentas; no parecen modificar los síntomas a mediano y largo plazo, por lo que resulta indispensable la implantación de una alternativa terapéutica integral que mantenga por tiempos prolongados la remisión de la sintomatología.

\section{Terapia multisistémica}

Es una herramienta terapéutica integradora, multimodal, que involucra tratamientos biológicos, psicosociales y psicoeducativos con una ambientación ecológica. Las características clínicas y principios para definir la TMS se apoyan en los correlatos de las conductas disociales, así como en la teoría social-ecológica de Bronfenbrenner, ${ }^{31}$ la cual concibe al individuo y a su comunidad como sistemas interconectados con influencias dinámicas y recíprocas. La TMS se orienta a la identificación de los problemas del adolescente y su familia en el contexto comunitario y sus intervenciones se realizan en el ambiente natural. Los objetivos están orientados a enriquecer a los padres con recursos y habilidades para contender con la crianza de adolescentes, y para que éstos hagan frente a los problemas familiares y extrafamiliares. La TMS utiliza diferentes estrategias familiares, individuales y farmacológicas para ayudar al adolescente; las intervenciones son individualizadas y altamente flexibles. Las acciones están apoyadas en la participación directa de los padres. Esta terapia implica a un profesional altamente capacitado y disponible para la familia las 24 horas del día. Las intervenciones familiares inicialmente pueden ser diarias y hasta semanales por tres a cinco meses. Existe un equipo de apoyo y monitoreo continuo de los objetivos, que se integra por psiquiatras, trabajadores sociales, psicólogos, pediatras, neurólogos, pedagogos y psicoterapeutas, entre otros.

\section{Principios de la terapia multisistémica}

1. El objetivo fundamental de la evaluación es comprender la forma en que interactúan los problemas identificados, y el contexto sistémico.

2. Los contactos terapéuticos destacan lo positivo y la fortaleza del abordaje sistémico para lograr el cambio.

3. Las intervenciones están diseñadas para promover entre los miembros de la familia las conductas responsables y disminuir las irresponsables.

4. Las intervenciones están enfocadas al presente y orientadas a la acción sobre problemas específicos y bien definidos.

5. Las intervenciones están orientadas secuencialmente hacia los diferentes sistemas que mantienen el problema.

6. Las intervenciones están diseñadas de acuerdo con el nivel de desarrollo del adolescente y satisface las necesidades de desarrollo del muchacho.

7. Las intervenciones están diseñadas para contar con esfuerzo diario o semanal de la familia.

8. La efectividad de las intervenciones se evalúa constantemente desde diferentes perspectivas, lo que permite superar barreras.

9. Las intervenciones están diseñadas para promover la generalización del tratamiento y para mantener los cambios terapéuticos a largo plazo, al tiempo que refuerzan a los cuidadores para las necesidades de los miembros de la familia en los diferentes contextos del sistema. 


\section{Estrategias en la implantación de los principios}

Principio 1. En la evaluación de los problemas y del contexto es importante desarrollar un listado de las fortalezas y las debilidades o problemas del adolescente en cada uno de los diferentes sistemas: el individual, el familiar, el de los compañeros, el de la escuela y el de la comunidad; una vez establecido el diagnóstico, el especialista deberá desarrollar una hipótesis conjuntamente con los miembros del equipo y el supervisor, teniendo en cuenta que todos los constructos o factores relevantes deben ser concretos y medibles; la hipótesis debe ponerse a prueba al evaluar los efectos de las intervenciones derivadas de la misma.

Principio 2. Para lograr que se mantenga el clima positivo de fortaleza y de cambio el clínico debe compartir esta actitud optimista y la expectativa con el equipo de trabajo, y transmitirlo de forma realista en todo contacto terapéutico; existen algunas acciones concretas que ayudan a mantener la fortaleza: primero, no utilizar términos peyorativos al hablar o escribir sobre el paciente, por ejemplo, los y las adolescentes no se ven "resistentes al tratamiento", sino que muestran un "desafío al tratamiento"; segundo, cuando el clínico tenga problemas para identificar aspectos positivos en el adolescente o en la familia deberá solicitar apoyo del equipo; tercero, el clínico debe enseñar técnicas para visualizar positivamente las situaciones o conductas y cuarto, el clínico debe mantener una actitud continua de búsqueda de soluciones, ofrecer una esperanza racional de mejoría y buscar y destacar lo que la familia hace bien.

Principio 3. Muchos padres se preguntan cuáles son las responsabilidades que deben tener sus hijos, y parece existir un acuerdo: respetar las reglas familiares y sociales, asistir a la escuela y esforzarse de manera razonable, ayudar en la casa y no dañar a otros.

Principio 4. Al orientar a la familia hacia el logro de los objetivos y enseñarles esta técnica se busca que ésta y sus integrantes logren generalizarla; es importante categorizar los objetivos que se pueden alcanzar a corto, mediano y largo plazo, y las acciones que deben guiar al adolescente y la familia a la solución de los problemas.

Principio 5. Al ser secuenciadas las intervenciones se requiere de una elevada individualización para cada familia y se busca, por medio de las transacciones interpersonales, que ésta y sus miembros puedan vincularse con los diferentes sistemas.

Principio 6. Las intervenciones deben adaptarse a la edad del niño o del adolescente con problemas disociales; para los niños y los adolescentes jóvenes se deben hacer importantes esfuerzos con el fin de aumentar el control de los padres; para los adolescentes mayores las intervenciones estarán orientadas a prepararlo para ingresar en el mundo de los adultos. Principio 7. El diseño de las intervenciones debe contener actividades diarias o semanales para cada miembro de la familia; el clínico se debe reunir cuantas veces considere necesario, hasta una diaria y, paulatinamente de acuerdo con el plan específico de cada familia, se retira del contacto, dando libertad gradual a la familia, pero permitiendo su eventual localización en caso de emergencias (radiolocalizador, teléfono celular, pager, etcétera).

Principio 8. Cuando las intervenciones han sido bien diseñadas pueden empezar a dar resultados después de pocas semanas. Múltiples informantes y múltiples métodos deben ser utilizados para evaluar las intervenciones; se deben cuidar dos aspectos al seleccionar al informante para la evaluación: confiabilidad y validez de la información.

Principio 9. La perspectiva del clínico involucrado en la TMS debe estar dirigida a que la familia pueda mantener los cambios logrados; algunas alternativas pueden ser enseñar las conductas o habilidades relevantes en el lugar donde normalmente experimenta la conducta el o la adolescente, entusiasmar y reforzar el desarrollo de habilidades que resuelvan problemas, encontrar a individuos en el medio comunitario del adolescente que refuercen las nuevas conductas de los miembros de la familia, hacerle ver a los demás significativos (maestros, familiares, etcétera) los cambios logrados y ofrecer un reforzamiento positivo cuando ocurra la generalización. ${ }^{32}$

Algunos de los aspectos relacionados con el éxito de la TMS exigen de quienes la implantan el estar completamente relacionados filosófica y empíricamente con ésta, tener un entrenamiento intensivo y una dedicación total, además de contar con apoyos e incentivos institucionales. Esta metodología terapéutica requiere iniciativa y colaboración de diferentes instituciones. Los resultados en el nivel de la delincuencia común señalan que es más efectiva que las intervenciones generales en la disminución de los problemas de conducta en adolescentes, disminuye su asociación con otros infractores y mejora las relaciones familiares, en especial la comunicación y el afecto. En el nivel de los agresores violentos o crónicos la TMS ha mostrado superioridad sobre las intervenciones convencionales o individuales, con menos arrestos, días de encarcelamiento, abandonos y dinero gastado, tanto a corto como a largo plazo (seguimiento a cuatro años). Además, disminuye más el número de detenciones por 
consumo de drogas y el uso de drogas "ligeras" que el tratamiento convencional. Es dinámica y, en constante innovación, ha mostrado también su utilidad en agresores sexuales. Su éxito estriba en su atención multifocal orientada a los agentes relacionados con las conductas disociales. El contexto de su intervención es natural y no creado en un centro de readaptación o de tutores, y busca cambios a largo plazo. ${ }^{33}$

\section{Investigaciones sobre la terapia multisistémica}

Recientemente Henggeler y su equipo de la Universidad de Carolina del Sur, en Charleston, Estados Unidos de América, publicaron una investigación donde utilizan la TMS como una alternativa a la hospitalización de jóvenes con crisis psiquiátricas. ${ }^{34} \mathrm{El}$ objetivo fue determinar si podía ser utilizada como una alternativa clínica viable a la hospitalización psiquiátrica. Se evaluó a 116 niños y adolescentes que llegaron a la unidad de urgencias del hospital universitario; aproximadamente $25 \%$ de la muestra cursaba con diagnóstico principal de trastorno disocial y fueron asignados de forma aleatoria a TMS basada en el hogar o a hospitalización. La evaluación de la sintomatología incluyó conductas disociales, autoestima, relaciones familiares, relaciones con los iguales, asistencia a la escuela y satisfacción con el servicio; se hicieron tres evaluaciones de estos parámetros: a las 24 horas, a las dos semanas y a los cuatro meses. La TMS fue más efectiva que la hospitalización en la reducción de los síntomas exteriorizados y mejoró más el funcionamiento familiar y la asistencia a la escuela, además de dar mayores calificaciones en la satisfacción con el servicio; la hospitalización fue más efectiva que la TMS para mejorar la autoestima. Estos hallazgos apoyan el hecho de que un modelo de tratamiento intensivo, bien especificado, con soporte empírico y con acceso al ambiente comunitario y familiar de adolescentes puede servir como una alternativa terapéutica a la hospitalización psiquiátrica. No existen reportes en la literatura internacional sobre el desarrollo de la TMS en territorio mexicano, lo que constituye un reto para el desarrollo de opciones de tratamiento en adolescentes disociales dentro y fuera del ambiente de los centros de rehabilitación social.

\section{Conclusiones}

El trastorno disocial es una categoría médico psiquiátrica que se caracteriza por una transgresión de las normas y derechos de los demás; su inicio antes de los 10 años de edad está relacionado con un mal pro- nóstico. Dentro de los elementos etiológicos del TD participan variables biológicas, psicológicas y sociales que lo determinan de forma significativa y que deben ser consideradas para su tratamiento. Hasta ahora ningún tratamiento individual ha servido en la reducción a largo plazo de los síntomas disociales en adolescentes con este padecimiento; por consiguiente, resulta indispensable la utilización de una herramienta terapéutica que involucre al paciente, a la familia y al contexto ecológico en la reducción de las manifestaciones antisociales. La TMS parece contar con las peculiaridades necesarias para la atención integral de adolescentes con más graves problemas de conducta: los que roban, los que asesinan, los que violan. Aunque existen algunas experiencias en la implantación de esta opción terapéutica es necesario desarrollar nuevas investigaciones que determinen a largo plazo los beneficios de la misma, en las cuales deberán participar las instituciones de salud involucradas, las dependencias del gobierno (como el Consejo Tutelar) y las instituciones privadas que puedan financiar estas empresas.

\section{Referencias}

1. American Psychiatric Associatión. Manual Diagnóstico y Estadístico de los Trastornos Mentales. Cuarta edición (DSM-IV). American Psychiatry Press, W ashington DC, 1994

2. O rganización Mundial de la Salud. CIE 10. Trastornos Mentales y del Comportamiento, Descripciones Clínicas y Pautas para el Diagnóstico, O MS, Ginebra, Suiza, 1992.

3. Hinshay SP, Anderson CA. Conduct and O ppositional D efiant Disorders. En: EJ Mash, RA Barkley, ed. Chld Psychopathology, The Guillford Press, N ueva York, 1996:113-149.

4. De la Peña F. Importancia clínica de la edad de inicio en el trastorno de conducta. Información Clínica, Instituto N acional de Psiquiatría Ramón de la Fuente M, 1999:10:5-6.

5. Lahey BB, Loeber R, Q uay H.Validy of DSM-IV subtipes of conduct disorder based on age of onset.J A m A cad C hild A dolesc Psychaitry 1998, 37:435-442.

6. Steinher $H$, Stone LA.Violencie and related psychopatology. J Am Acad Child Adolesc Psychiatry 1999:38:3-5.

7. Scott CL. Juvenile violence. Psychiatric Cli N orth Am 1999, 22:71-83. 8. De la Peña F.Violencia Juvenil. Información C línica, Instituto $\mathrm{N}$ acional de Psiquiatría Ramón de la Fuente M, 1999, 10:33-35.

9. De la Peña F, Patiño M, Mendizábal A, C ortés J, C ruz E, U lloa RE et al. La entrevista semiestructurada para adolescentes (ESA). C aracterísticas del instrumento y estudio de confiabilidad interevaluador y temporal. Salud Mental 1998 21:11-18.

10. Kaufamn J, Birmaher B, Brent D, Rao U. Schedule for affective disorders and schizophrenia for school age children-presente and lifetime version (K-SADS-PL): Initial reliability and validity data.J A m A cad C hild A dolesc Psychiatry 1998;36:980-988.

11. Edelbrok C. Child behavioral check list (CBCL). Psychopharmacology Bull 1985; 21:835-839.

12. Conners CK. Parent symptom questionare. Psychopharmacology Bull, 1985:816-823. 
13. Kaminer Y. Addictive disorders in adolescents. Psychiatric Cli N orth Am 1999;22:275-288.

14. Villatoro J, Medina-Mora ME, C ardiel H. La situación del consumo de sustancias entre estudiantes de la Ciudad de México. Salud Mental 1999; 22:18-30.

15. De la Peña F. Las drogas y los adolescentes. Información C línica, Instituto N acional de Psiquiatría Ramón de la Fuente M, 1999;10:56-57.

16. Pihl Ro, Hoakenn PNS. Clinical correlates and predictores of violence in patients with substance use disorders. Psychiatric Annals 1997; 27: 735-740.

17. González JA. Correlaciones clínicas y predictores de violencia en pacientes con trastornos por abuso de sustancias. Información Clínica, Instituto N acional de Psiquiatría Ramón de la Fuente M, 1999;10:26-27.

18. Sholevar GP, Sholevar EH. O verview. En: G. Pirooz Sholevar, ed. Conduct disorders in children and adolescents. Am Psychiatric Press, W ashington DC, 1995: 3-27.

19. Lewis D O. Conduct disorder. En: Melvin Lewis, ed. Child and adolescent psychiatry a comprehensive textbook, second edition, W illiams \& W ilkins, Baltimore, MD 1996:564-576.

20. Malone RP, Pena-Ariet M, Biesecker K, D elaney MA. The over aggression scale in a study of lithium in aggressive conduct disorder. Psychopharmacol Bull 1994; 30: 215-218.

21. Campbell M, Adams PB, Small AM, Kafantaris V, Silva RR, Shell J et al. Lithium in hospitalized aggressive children with conduct disorder: A double-blind and placebo controlled study. J A m A cad C hild A dol Psych 1995; 34:445-453.

22. Geller B, Cooper TB, Sun K, Zimerman B, Frazier J,W illiams M. D ouble-blind and placebo control study of lithium for adolescent bipolar disorders with secondary substance dependency. J Am Acad Child Adolesc Psychiatry 1998;37:171-178.

23. Heiligenstein JH, Beasley CM JR, Potvin JH. Fluoxetine not associated with increased aggression in controlled clinical trials. Int C lin Psychopharmacol 1993; 8:277-280.
24. C onstantino JN , Liberman M, Kincaid M. Effects of serotonin reuptake inhibitors on aggressive behavior in psychiatrically hospitalized adolescents: Results of an open trial. J Child Adolesc Psychopharmacol 1997; 7:31-44.

25. C owdry R, Gardner D. Pharmacology of borderline personality disorder.Arch Gen Psychiatry 1988; 45:111-119.

26. Almeida LG, De la Peña F. La risperidona como alternativa farmacológica en el trastorno bipolar de inicio en la infancia y en la adolescencia. Información C línica Instituto $\mathrm{N}$ acional de Psiquiatría Ramón de la Fuente M, 1999; 10:59-60.

27. Fraizer JA, Meyer MC, Biederman J. Risperidone treatment for juvenile bipolar disorder: A retrospective chart review. J Am A cad Child Adolesc Psychiatry 1999; 38:960-965.

28. Connor DF, Fletcher KE, Swanson JM.A meta analysis of clonidine for symptoms of attention deficit hyperactivity disorder. J Am Acad Child Adolesc Psychiatry 1999; 38:1551-1559.

29. Green W H, ed. Child and adolescent clinical psychopharmacology, second edition, W illiams \& W ilkins, Baltimore MD, 1995.

30. Hibss DE,ed. Psychosocial treatment for child and adolescent disorders. A merican Pscychological Association, W ashington DC , 1996.

31. Bronfenbrenner U.The ecology of human development. Harvard University Press, Cambridge MA, 1979.

32. Barlow $D$, ed. Multisystemic treatment of antisocial behavior in children and adolescents. First edition, The Guilford Press, NY, 1998.

33. Borduin CM, Multisystemic treatment of ciminality and violence in adolescents. J Am Acad Child A dolesc Psychiatry 1999; 38:242-249.

34. Henggeler SW, Rowland MD, Randal J, W ard DM, Pickrel SG. Homebased multisystemic therapy as an alternative to the hospitalization of youths in psychiatric crisis: Clinical outcomes. J Am Acad Child Adolesc Psychiatry 1999;38:1331-1339. 